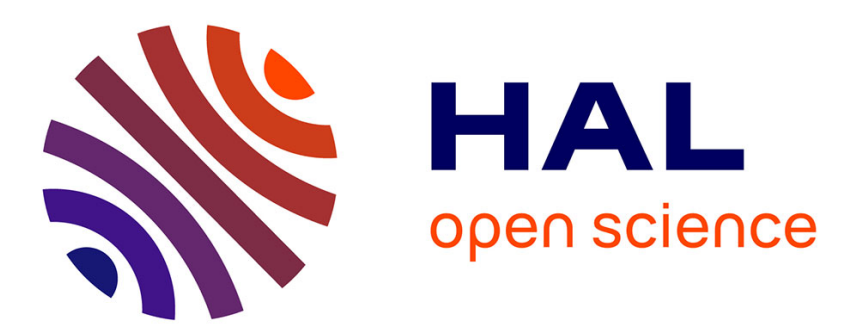

\title{
An optimized use of limited ground based topographic data for river applications
}

\author{
M. Jaballah, B. Camenen, André Paquier, M. Jodeau
}

\section{To cite this version:}

M. Jaballah, B. Camenen, André Paquier, M. Jodeau. An optimized use of limited ground based topographic data for river applications. International Journal of Sediment Research, 2019, 34 (3), pp.216-225. 10.1016/j.ijsrc.2018.11.002 . hal-02608046

\section{HAL Id: hal-02608046 https://hal.inrae.fr/hal-02608046}

Submitted on 16 May 2020

HAL is a multi-disciplinary open access archive for the deposit and dissemination of scientific research documents, whether they are published or not. The documents may come from teaching and research institutions in France or abroad, or from public or private research centers.
L'archive ouverte pluridisciplinaire HAL, est destinée au dépôt et à la diffusion de documents scientifiques de niveau recherche, publiés ou non, émanant des établissements d'enseignement et de recherche français ou étrangers, des laboratoires publics ou privés. 


\title{
An optimized use of limited ground based topographic data for river applications
}

\author{
Mohamed Jaballaha ${ }^{\mathrm{a}}$ Benoît Camenen ${ }^{\mathrm{a}, *}$, André Paquier ${ }^{\mathrm{a}}$, Magali Jodeau \\ ${ }^{a}$ Irstea, UR RiverLy, centre de Lyon-Villeurbanne, 5 Rue de la Doua, CS 20244, \\ F-69625 Villeurbanne cedex, France \\ ${ }^{b}$ EDF RED, 6 quai Watier - 78401 Chatou cedex, France
}

\begin{abstract}
A prime requirement for hydrological applications, such as sediment budgeting or numerical modelling, is that produced Digital Terrain Models (DTMs) accurately represent the shape of landforms, especially for river reaches where data are not homogeneous. DTM error is a function of data point measurement accuracy and density and also of the field survey strategy when limited amounts of data will be acquired. This paper aims to advance the importance of the field survey strategy for the specific, but common cases, where only limited topographic data will be available. This methodology is based on the idea that any feature can be properly described by a set of cross sections and breaklines describing both main and secondary directions of the flow. Then, a longitudinal linear interpolation can be applied to the defined homogeneous zones. This morphologically oriented (MO) method that includes data acquisition strategy and interpolation, was validated using a reference DTM derived from LiDAR measurements. An estimation of the uncertainties also is suggested based on the distance of the nearest point and the local slope using a geographically weighted regression. The proposed MO method is typically applicable to Alpine river reaches characterized by multiple channels that may always be underwater and not navigable such as an alternate bar system with secondary and transverse channels.
\end{abstract}

Keywords: Digital Terrain Model, Survey strategy, Interpolation, DTM

\footnotetext{
${ }^{*}$ Corresponding author, Tel: +33(0)472208607, Fax: +33(0)478477875

Email addresses: jaballah.med@gmail.com (Mohamed Jaballah), benoit.camenen@irstea.fr (Benoît Camenen), andre.paquier@irstea.fr (André Paquier), magali.jodeau@edf.fr (Magali Jodeau)
} 
error, Gravel bar

\section{Introduction}

Accurate river bed topography reproduction is a key factor for hydraulic and sediment transport understanding and modelling. Merwade et al. (2008) and Schäppi et al. (2010) emphasize the importance of detailed and accurate river bathymetry in hydraulic modelling especially to create robust two/three-dimensional (2D/3D) hydrodynamic models. Furthermore, Digital Terrain Models (DTMs) have become a key tool in recent fluvial geomorphology research to assess and quantify morphological changes and sediment budgets using repeated topographic surveys (Brasington et al., 2000; Carley et al., 2012; Fuller et al., 2003; Legleiter \& Kyriakidis, 2008; Wheaton et al., 2010).

While traditional approaches for one-dimensional (1D) modelling applications describe the river bathymetry by the mean of several cross-sections (e.g. Aggett \& Wilson, 2009), 2D/3D approaches and geomorphologic studies require more detailed topographic information (Lane, 1998; Legleiter et al., 2011). Extensive literature are available on the application of hydraulic modelling to assess hydraulics or sediment transport in rivers (Horritt \& Bates, 2002; Lane \& Ferguson, 2005, etc.).

DTMs can be developed by different methods depending on data collection techniques (Erdogan, 2009): (i) field surveying: interpolation of existing cross sections and/or interpolation of discrete bathymetry points collected using echo sounding techniques, total-station theodolite or differential Global Positioning System (dGPS); (ii) satellite-based and/or airborne techniques: Light Detection and Ranging (LiDAR); (iii) digital photogrammetry, including Structure-from-Motion (SfM, Westoby et al., 2012). LiDAR and digital photogrammetry have the great advantage to provide dense and spatially distributed information but generally limited to surface elevation as no information is provided under water nor under vegetation for photogrammetry (Brasington et al., 2003; Legleiter, 2012). Recent through-water photogrammetry and bathymetric LiDAR are capable to measure the channel bed but only for clear shallow waters and with larger uncertainties (Woodget et al. 2015).

In the case of Alpine rivers, a significant part of the river bed is always under water (primarily the main channel), and water colour generally is grey 
due to the high concentrations of sediment. As a consequence, the river bed cannot be described by the afore mentioned methods. The ground-based methods using dGPS and total station theodolites have many advantages such as providing accurate point measurements of bed elevation under the water surface, the ability to select measurement points describing micro-forms and breaks in slope, adaptability to a pre-defined strategy, and finally ease of access and relatively low cost, compared to some of the other alternatives, allowing these methods to be suitable for multiple surveys (Bangen et al. 2014). Nevertheless, collection of river topography data using ground-based technique is a labour intensive and money-consuming task. Such surveys also are limited to wadable reaches with relatively limited vegetation.

Various interpolation methods of sparse topographic measurement points yielding DTMs, which are regular grid representation of surface curvature, are used and explained in multiple studies, e.g., Delaunay Triangulation (DT), Inverse Distance Weighted (IDW) function, Kriging, local polynomial, spline, etc. Erdogan (2009) relates DTM quality upon three main factors: (i) accuracy, density, and distribution of the source data; (ii) the interpolation process; and (iii) the characteristics of the surface. In the case where just low density and sparse elevation data points are available, a straightforward triangulation will likely be misleading and generate an inaccurate DTM due to artefacts. One should have in mind that measured data and grid resolution must be set to capture the smallest surface features the study is targeting.

Applying a robust surveying methodology is crucial. Keim et al. (1999) explain in detail a methodology for topographic surveying of mountain streams using a total-station theodolite and stress the importance of the use of breaklines in the DTM processing using the Triangular Irregular Network (TIN) interpolation algorithm. A breakline can be defined as a topographic break in slope in a cross section such as a bank top, toe slope, or thalweg. In most studies (French \& Clifford, 2000, Keim et al., 1999, Schäppi et al., 2010, etc.) breaklines are, however, defined along the channel only. Heritage et al. (2009) showed that the greatest vertical error is likely to be found at breaks of slope. They also highlighted the limitation of a method using cross sections only and suggested a morphological method based on a description of bar and chute outlines.

The aim of the current paper is to provide a simple morphologically oriented (MO) method along with elevation error estimation to effectively monitor river bed topography in order to create DTMs. The methodology presented here is a development of previous work by optimizing the ground- 
based survey. The methodology emphasizes the construction of breaklines to describe the flow in both main and secondary channels, including transverse channels. It combines the method based on cross sections and breaklines and the morphologically-based method as introduced by Heritage et al. (2009). Based on a limited amount of data, it is intended to produce a DTM suitable for a simple and straightforward use for both morphological assessment and budgeting as well as 2D hydraulic modelling. This method is proposed primarily for Alpine river channels, which are always under water and wadable only during low flow periods and where typical features such as islands or gravel bars can be observed. At the moment, topographic, ground-based techniques remain the most suitable choice to measure bathymetry in these river systems. The MO method was applied in a reach of the Arc River in the French Alps and consists of: (i) acquiring data as cross sections of both the main channel system and secondary features, which are supported by a set of discrete point measurements describing breaks in slope and unusual morphological forms, such as bar limits and transverse channels; (ii) interpolating by the mean of an adaptive interpolation method to reproduce topographic microforms; and (iii) an assessment of the interpolation error. For the interpolation error, an estimation of the uncertainties is suggested based on the distance of the nearest point and the local slope using a geographically weighted regression.

The current paper is organized as follows. The first part addresses the development of the MO method where a case field study is illustrated. In the second part, different DTM error sources are presented. The third part shows results of the $\mathrm{MO}$ validation using a LiDAR derived DEM as a reference data set. Finally, uncertainty estimation is introduced and discussed.

\section{Methodology}

\subsection{Study site}

The study site is a specific gravel bar within a system of alternate bars located in the Arc River (France). It is the subject of a large comprehensive study including field monitoring and flow and sediment measurements in order to assess qualitatively and numerically the morphodynamics of Alpine rivers and gravel bars as a case study (Jaballah, 2013; Jodeau, 2007). Thus, field surveys have been done several times per year from 2005 to 2013. It includes a $400 \mathrm{~m}$ long bar located on the left bank. The river width $(W)$ is approximately $60 \mathrm{~m}$, the mean local slope $S$ is approximately $0.6 \%$, and the bed 
sediment is composed of a mixture of gravel (median diameter $D_{50} \approx 0.05 \mathrm{~m}$ ) and fine sediment (Camenen et al., 2010). The study site is a good example for the MO development since it is a well documented site and it includes a typical bed macro-form (gravel bar) widely observed in mountainous regulated rivers (Fig. 1). The gravel bar is out of the water most of the time (except for relatively high discharges corresponding to a one-year return period flood). The study reach includes a secondary channel on its left side of the bar. When water inundates the bar, it carries a significant part of the flow to the connecting secondary channels. Thus, two transverse channels connecting the secondary channel to the main channel exist at the middle part of the bar (Fig. 1). A third transverse channel is formed in the downstream part of the bar due to the drainage system outflow of the nearby highway. The main role of transverse channels consists of transferring water from the secondary channel to the main channel at the right side of the reach.

\subsection{Data collection: survey strategy}

The dGPS system (using a known reference station) and a total-station theodolite were used for digital topographic data collection with a low three dimension uncertainty (1 to $5 \mathrm{~cm}$, see Brasington et al., 2000, MiroslawSwiatek et al., 2016). The configuration of the reach and the time and labour limitations on field work influenced the selected methodology for data acquisition. Assuming that the river shape results from its flow intensity and that flow direction plays a fundamental role in modulating the river bed, point measurements have been taken in three steps to describe all main morphological features.

- First, a set of full cross sections (linking the two dykes across the reach) was measured every $20 \mathrm{~m}$ approximately $(\approx W / 3)$ in the longitudinal direction of the river using a total-station theodolite. An interpolation of surveyed cross sections will lead to a reproduction of the main reach topographic aspects: main channel, secondary channel, and the bar surface.

- Second, for each transverse channel, a new set of cross sections is measured, e.g., each transverse channel was considered as if it stands as a separate river channel.

- Third, additional topographic point measurements detecting breaks in slope and the bar outline were acquired to enrich the dataset. 


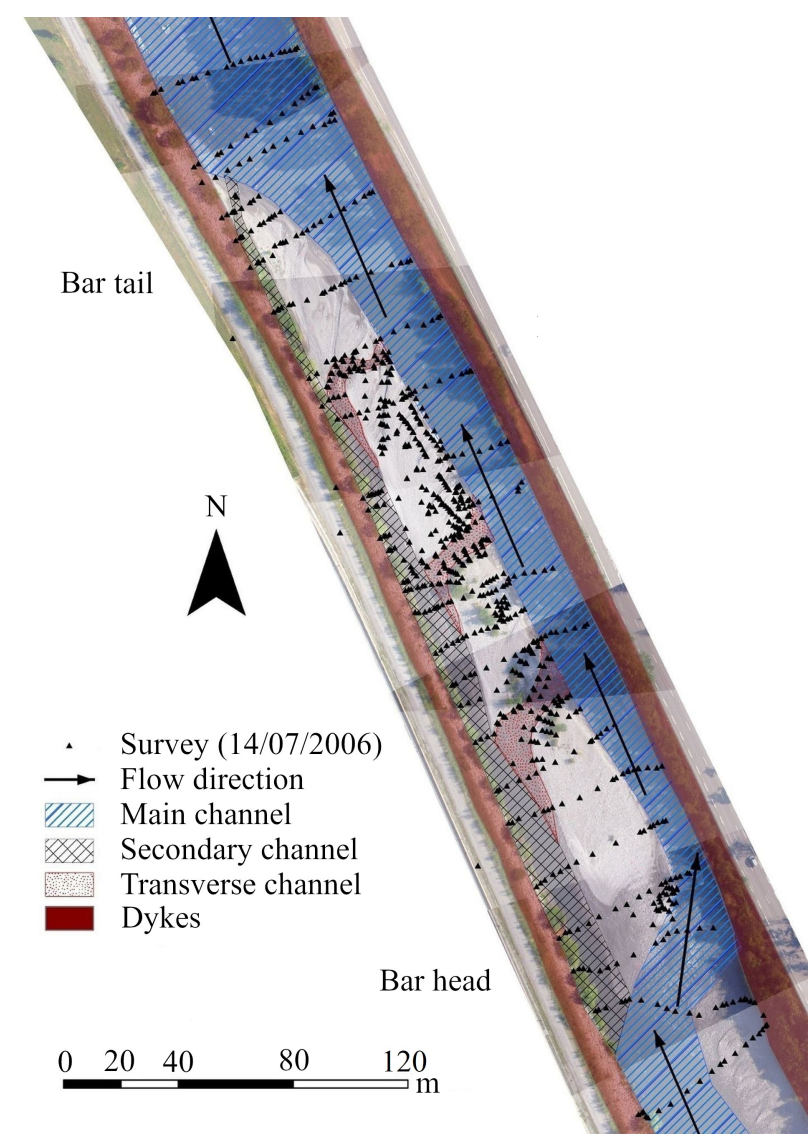

Fig. 1. Bar components and dispersion of topographic point measurements. An assembly of multiple aerial photos of the site, taken on the same date, is shown in the background.

Finally, a set of control permanent points was established to define required geographic transformations and allow an inter-comparability of different field campaigns.

\subsection{Interpolation method and steps}

The mean point density of field campaigns is around 0.04 points $/ \mathrm{m}^{2}$. This data density can be considered low compared to the channel length and its features scales, but it can be sufficient to describe the reach if a proper interpolation method is applied. The study site presents two major characteristics with different digital elevation data densities: the main channel and the bar surface. While the mean longitudinal space lag between two successive full 
cross sections is $20 \mathrm{~m}$, this spatial lag drops to a few meters on transverse channels. This difference addresses the issue of grid resolution selection.

The other key issue is connected to interpolation direction and how to interpolate cross sections, which are perpendicular to the main flow direction (e.g., transverse channels). Hence, the choice of an interpolation method is crucial.

The following steps were defined and followed to interpolate elevation data onto a regular grid (Fig. 2):

(i) A point dataset describing the two dykes is fixed (dykes made of boulders are stable, therefore, the same data can be used for several surveys).

(ii) Full cross sections are first set following field measurements. Streamwise breaklines describing the main channel, the secondary channel, and the bar limits are defined in order that no break in slope can be detected in the transverse direction inside a given zone limited by two successive breaklines. Following Keim et al. (1999), breaklines are defined on bank tops, bank toes, and the thalweg. Cross section visualization is pre-required for the identification of numerous breaklines since many of them cannot be distinguished from the scatter of points by just using an aerial photo as the background. FUDAA-MODELEUR software (https://sourceforge.net/projects/fudaa/files/fudaa-mox/), a program intended to pre-process geometric data for many hydraulic models is used to do this step (Pénard, 2010).

(iii) A first linear interpolation following breaklines is done using SECMA, an interpolation tool developed at Irstea that allows many interpolation options and methods (Pénard, 2010).

(iv) Cross sections forming the secondary channels and incomplete cross sections are defined from point measurements. Since true point measurements in this case do not reach the two bordering dykes, cross sections were completed using the interpolated points from step (iii) to represent the whole transversal distance of the reach. Therefore, the newly defined set of complete cross sections incorporates a whole description of various surface topography features. True point measurements reflect transverse channels and abrupt breaks in slope and interpolated points represent the main channel. 
(v) Cross sections defined in the previous two steps are regrouped together in the same file. New breaklines are defined carefully to describe all morphologic units: main channel, bar limits, and secondary and transverse channels (Fig. 2b). Using SECMA, a second linear interpolation with respect of the breaklines is applied to form a new scattered point dataset over an irregular grid where the mean cell size is $0.5 \times 0.5 \mathrm{~m}$.

(vi) All other measured points not used yet are incorporated in the final interpolation using another tool called MODIFM that looks for matching points and modifies the elevation data by measured values.

A survey done on 24 October 2011 was taken as an example to illustrate the proposed interpolation method (Fig. 2d). Eventually, the final rendered DTM can be seen in Fig. 3. The DTM is visualized over a regular $0.5 \times 0.5 \mathrm{~m}$ grid using the triangulation with linear interpolation option in SURFER(C) software (Surfer, 2002). As can be seen in Fig. 3, all complex terrain surface topographies (bar edge, transverse channels, etc.) are properly reproduced in the rendered DTM. The authors in-house routines have been used in order to develop a DTM that can be used as a mesh for hydraulic modelling. Nevertheless, it is important to notice that the multiple routines used here are common functionalities implemented in many GIS software packages.

Heritage et al. (2009) introduced a morphologically-based method arguing that a method using cross sections leads to significant errors. However, they applied direct common interpollation procedures, which do not use breaklines or only major breaklines for the TIN approach (Delaunay triangulation with linear interpolation). The proposed approach combines a morphologicallybased method together with the use of breaklines defined using a set of cross sections. Moreover, the two-step methodology allows the description of transverse channels that could not be described by the first set of cross sections.

\section{Error and associated uncertainty estimation}

DTMs provide models of the continuous terrain's surface elevation based on point measurements, and, unfortunately, deviations from the true value of surface elevation cannot be avoided. This deviation is known as error. The lack of knowledge about these errors constitutes uncertainty (Wechsler, 2007). In the past decade, a considerable amount of research has been done to 
(a)

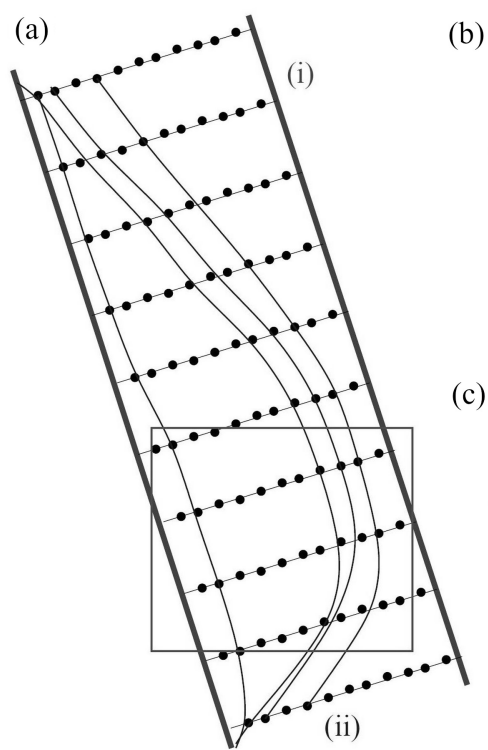

(b)

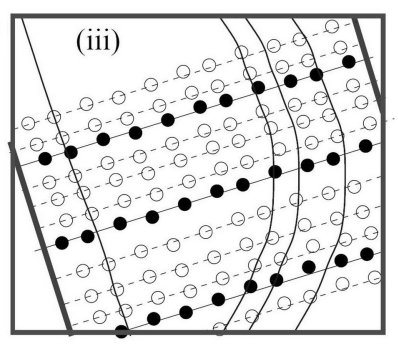

(c)

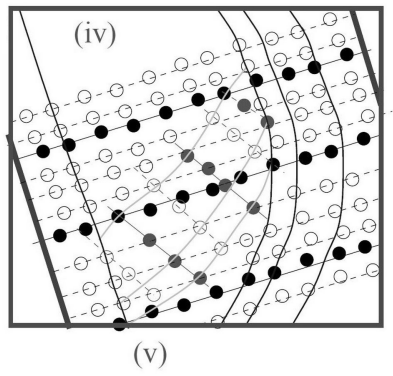

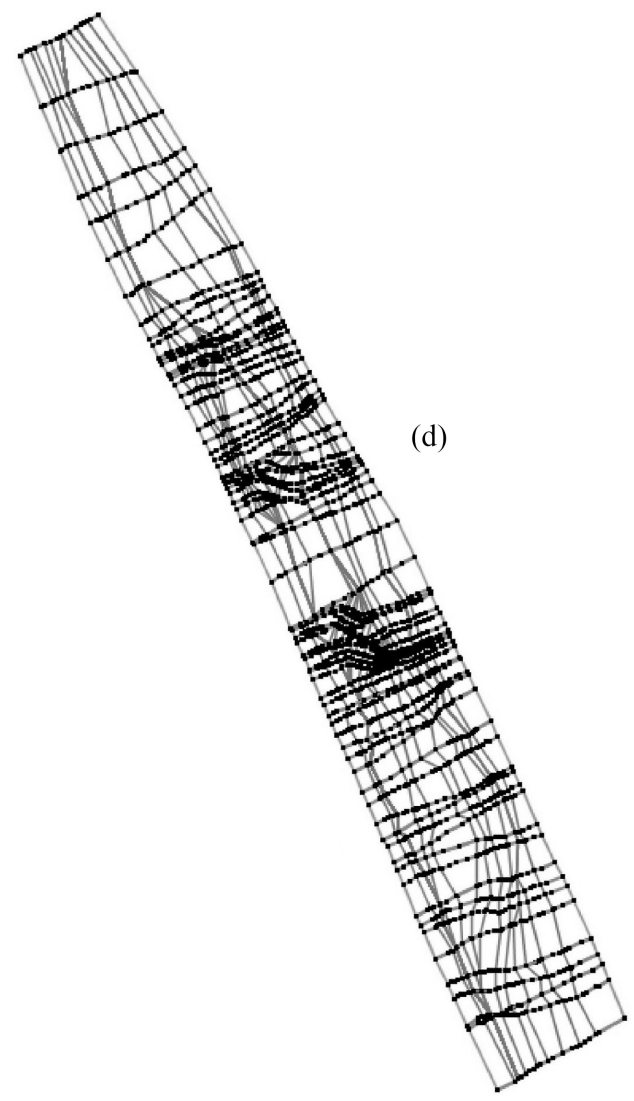

Fig. 2. Schematisation of the interpolation steps: (a) full cross sections, dykes, and major breaklines [Steps (i) and (ii)]; (b) interpolation [Step (iii)]; (c) final cross sections set and additional breaklines defining all topographic units [Steps (iv) and (v)]; and (d) results after step (v) for the 2011 dataset.

address the issue of DTM errors (Erdogan, 2009, Legleiter \& Kyriakidis, 2008; Milan et al., 2011; Wheaton et al., 2010, etc.). Main sources of error originate from a low measurement density and from an incorrect measurement strategy and are propagated through interpolation.

In the current paper only the elevation uncertainty will be considered and it is denoted as $\delta(z)$, which can be defined as follows:

$$
Z_{\text {real }}=Z_{\text {model }} \pm \delta(z)=Z_{\text {model }} \pm\left[\delta_{\mathrm{m}}(z)+\delta_{\mathrm{r}}(z)+\delta_{\mathrm{p}}(z)\right]
$$

Sources of uncertainties $(\delta)$ are mostly related to two steps in DTM con- 


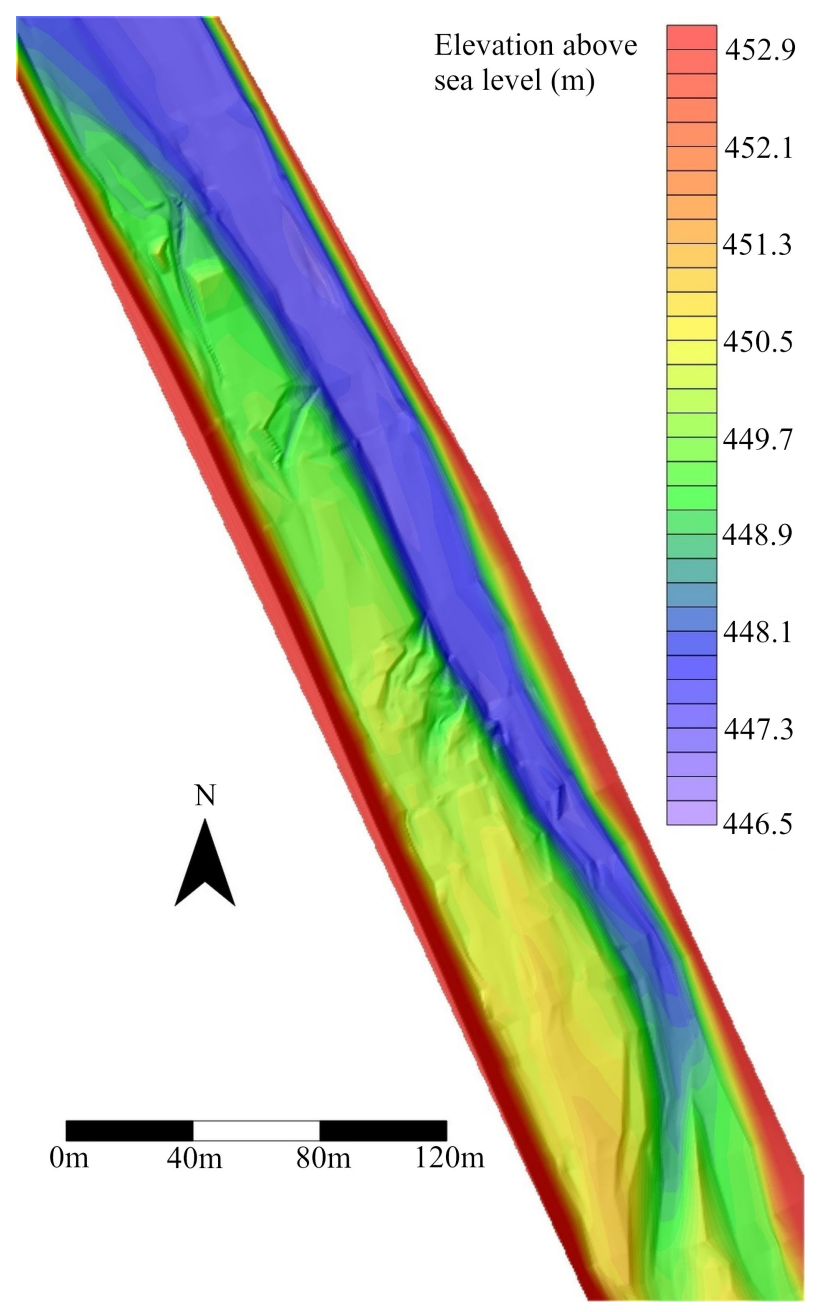

Fig. 3. DTM of the 2011 survey rendered by the MO interpolation method (a shaded relief map is shown behind the map for context).

struction: data acquisition and interpolation. Data acquisition generates two errors, which are linked to measurement errors $\left(\delta_{\mathrm{m}}\right)$ of devices used such as tachometers or dGPS and uncertainties due to the surface roughness $\left(\delta_{\mathrm{r}}\right)$. The other source of vertical error is due to the interpolation steps $\left(\delta_{\mathrm{p}}\right)$.

\subsection{Measurement and roughness error assessment}

Measurement errors are assumed to be constant here and taken as $\delta_{\mathrm{m}}=$ $0.02 \mathrm{~m}$; but these errors could be taken even smaller, e.g., Heritage et al. 
(2009) assumed $\delta_{\mathrm{m}}=0.005 \mathrm{~m}$. Surface measurement corresponds to the bias of sampling. In fact DTM production aims at reproducing the mean bed surface level. An operator in the field cannot assess this mean surface level. As shown in Fig. 4, in reality sampling points fall either above or below the targeted level. Therefore, there is a dependency on the bed roughness, especially in the case of gravel beds, where large sediment diameters can affect the measurement process.

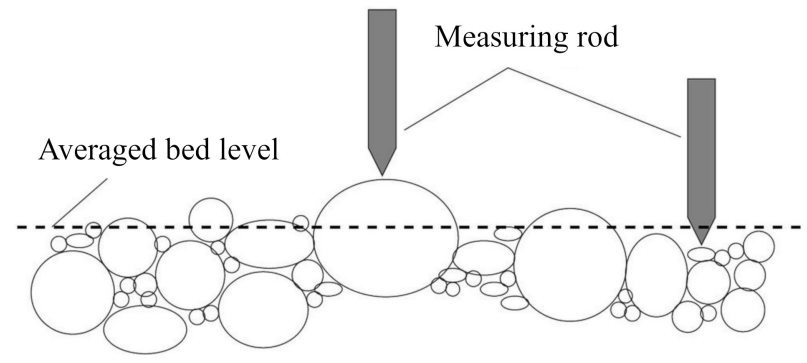

Fig. 4. Measurement error due to the bed roughness and operator choice: measuring rod lies somewhere above or below the targeted average bed level.

In research studies, it is common to compare error magnitude to the grain size of the experimental site assuming that uncertainty is strongly correlated to grain size (Brasington et al., 2000, Carlisle, 2005; Legleiter \& Kyriakidis, 2008). It is suggested that:

$$
\delta_{r}=\alpha D_{84}
$$

with $\alpha \approx 0.5$ and $D_{84}$ is the representative grain size for which $84 \%$ of the particles is finer. In the case of the Arc River bed surface, the geometric standard deviation $\sigma_{D}=\sqrt{D_{84} / D_{16}} \approx D_{84} / D_{50} \approx 2$ (assumption of a lognormal distribution of sediment grain sizes). Hence, the median diameter can be a good representation of the roughness error (i.e. $\delta_{r} \approx D_{50}$ ). The sediment grain-size spatial distribution is shown in Fig. 5. This grain-size map was obtained based on the Wolman (1954) sampling method (Jodeau, 2007) considering homogeneous zones. In this case, the mean diameter lies between $1 \mathrm{~mm}$ to $8 \mathrm{~cm}$ on the gravel bar.

\subsection{Interpolation error estimation}

Interpolation error is generated by the interpolation algorithm. Different surface interpolation approaches (e.g., Kriging, natural neighbors, TIN) lead to different elevation estimates. A comparison of interpolation methods for producing digital elevation models can be found in Erdogan (2009). 


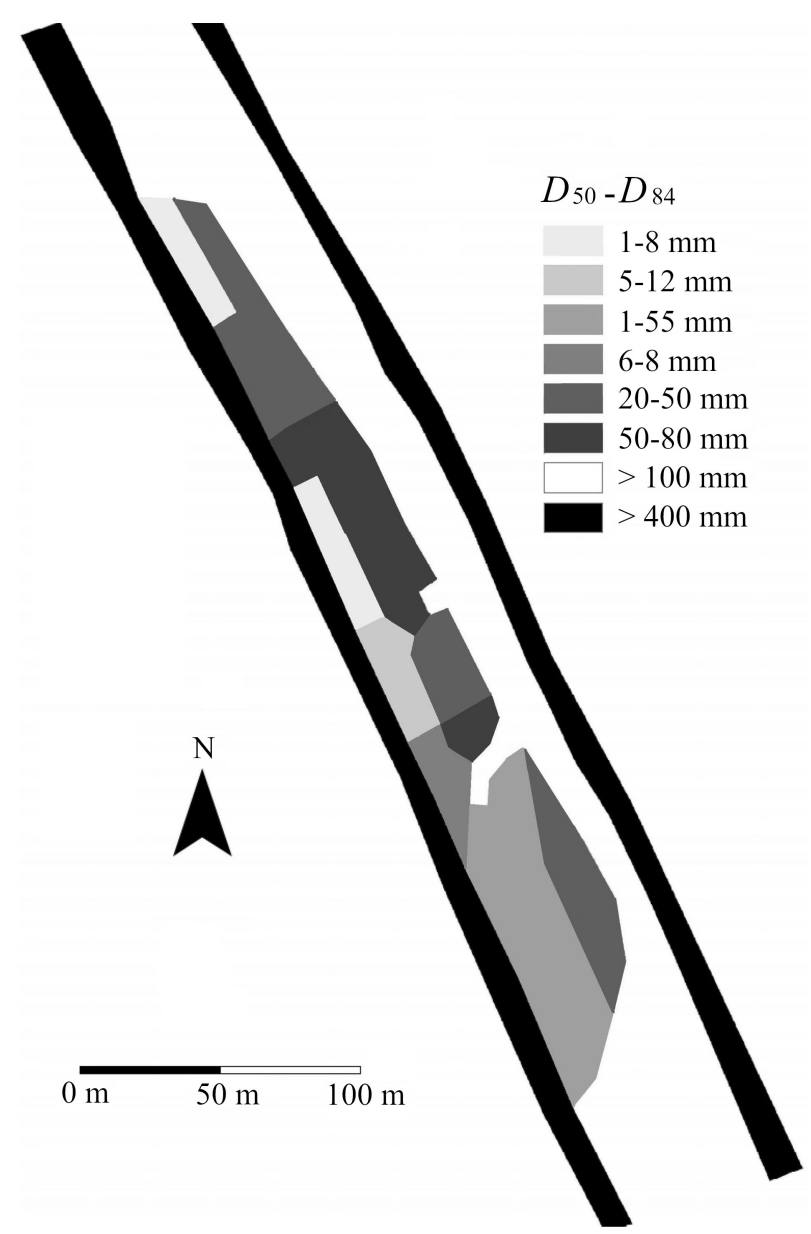

Fig. 5. Grain-size $\left(D_{50}\right.$ and $\left.D_{84}\right)$ spatial distribution over the gravel bar and main channel.

There is a general agreement that there is a significant correlation of DTM error and geomorphometric parameters (Desmet, 1997; Wechsler, 2007). Carlisle (2005) has used stepwise selection of variables to include in a multi variate Ordinary Least Squares (OLS) linear regression model. He was able to produce a well-fitted model, but that was after developing a multitude of predictor variables based on 12 topographic parameters. Erdogan (2010) has used a high resolution DTM to produce an interpolated DTM with coarser resolution. Then, he applied global statistic analysis, OLS regression, and Geographically Weighted Regression (GWR) developed by Fotheringham et al. (2002), to evaluate elevation error and calculate the spatial correlation to to- 
pographic parameters. His study concluded that the GWR method is more suitable and allows reproduction of spatial variable correlation coefficients unlike the OLS method.

Following the hypothesis that DTM accuracy is related to surface geomorphometric parameters, the absolute error introduced by the interpolation process was examined through the slope and distance to the nearest measured point. These terrain parameters are descriptive and are easily calculated from the computed DTM and field surveys. A Geographically Weighted Regression (GWR) was done in order to capture spatial heterogeneity in the relation between DTM error and geomorphometric parameters (Erdogan, 2010). The GWR is a local spatial statistical technique where local statistics are treated as a spatial disaggregation of global statistics (Fotheringham et al., 2002). The GWR tool implemented in ArcGIS 10.0 was applied and tested to relate the DTM error (absolute value of the difference between the real and interpolated elevations based on results from section 4.1) with multiple combinations of morphometric parameters. A Gaussian kernel function was used as adaptive with variable bandwidth size to provide a continuous weighting function up to a defined distance from the regression point (Erdogan, 2010). The optimum combination of explanatory variables leads to Eq. 3

$$
Z\left(x_{i}\right)=\alpha_{0}\left(x_{i}\right)+\alpha_{1}\left(x_{i}\right) d\left(x_{i}\right)^{-2}+\alpha_{2}\left(x_{i}\right)\left\|\overrightarrow{\operatorname{grad}}\left(x_{i}\right)\right\| .
$$

where $Z\left(x_{i}\right)$ is the error introduced by the interpolation process at location $x_{i}, d\left(x_{i}\right)$ is the distance of the nearest measured point to the interpolated point $x_{i},\left\|\overrightarrow{\operatorname{grad}}\left(x_{i}\right)\right\|$ is the slope at the interpolated point $x_{i}, \alpha_{0}\left(x_{i}\right)$ is the intercept parameter, and $\alpha_{1}\left(x_{i}\right)$ and $\alpha_{2}\left(x_{i}\right)$ are the correlation coefficients.

\section{Validation of the methodology}

Assessments of measurement and interpolation error are discussed for the specific case of the studied reach following the approach proposed by Erdogan (2010).

\subsection{Validation using airborne LiDAR}

The proposed validation is based on the application of the MO method on a reference data set. Creating hypothetical data sets can be a good way to produce a reference data set. However, to stay in the same configuration and at a similar level of topographic complexity, an Airborne LiDAR DTM of 
the same reach was selected as reference data. The reader should be aware that LiDAR data are used here as a reference hypothetical data set and this paper does not evaluate LiDAR data error or any other issue related to compare field data and LiDAR data. Airborne LiDAR data were collected in May 2010 when the water level surface was low compared to the early summer period. These data were acquired in their interpolated form as a grid of $0.25 \times 0.25 \mathrm{~m}$ with a vertical resolution estimated at $0.05 \mathrm{~m}$. The generated DTM is used as reference data, e.g., it was considered as a true terrain representation.

To apply the MO method, a sample of sparse points was picked from the reference data in the same way a field survey is performed with a relatively low density as can be seen in Fig. 6a (to be compared with Fig. 1). It should be noticed that the validation zone was delimited just to the bar surface without taking into account the main channel, which cannot be detected by LiDAR due to the very low reflection of light waves by water.

The MO interpolation methodology based on a limited number of cross sections was applied to recreate a DTM. Figure 6b1 shows the DTM of difference between the reconstructedDTM and the reference DTM. It is clear that terrain elevations produced by the MO lie within the reference data. The MO reproduces most elevations within an interval of $\simeq 0.10 \mathrm{~m}$. The amplitude of error is similar to the size of gravel observed in the studied reach. However, some small discrepancies exist between the modelled elevation values and reference values. The magnitude of these local differences is about $0.5 \mathrm{~m}$. By examining the reference DTM and aerial pictures, it is found that some of these local differences correspond to boulders and micro-channels present along the edge of the upstream part of the gravel bar. In fact, the edge at this part of the bar is very steep and numerous breaches were formed which can be assimilated to form micro-channels. Thus, differences in the DTMs can be explained by the ability of LiDAR to cover more regularly the whole reach zone, while MO cannot yield a similar coverage. Overall, it can be concluded that MO is efficient and recreates the main topographic characteristics of the reference DTM with minor differences, which are localized and clustered. Consequently, the MO method is robust enough to capture a certain low level of terrain features regardless some microforms, which are considered to have little impact in either hydraulic and/or morphological studies. 
(a)

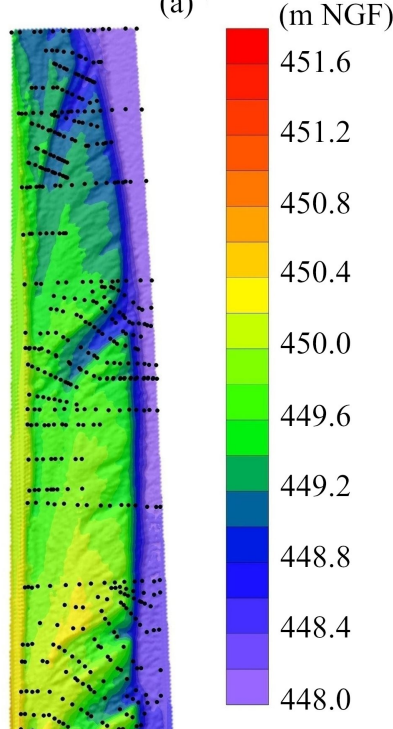

(b1)

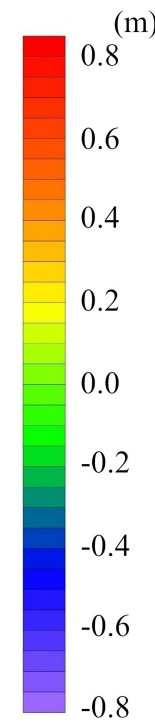

(b2)

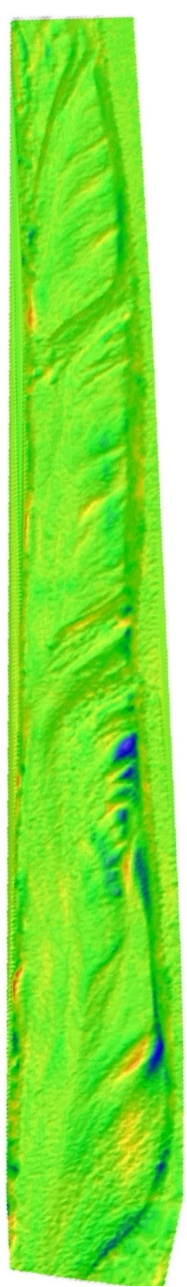

(b3)

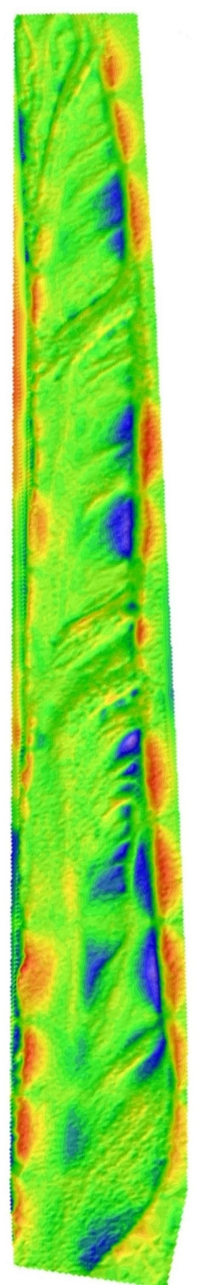

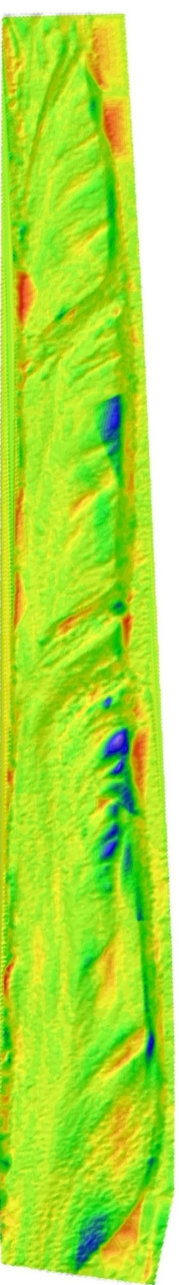

Fig. 6. Scatter of points chosen from the reference LiDAR DTM in $m$ Nivellement Général de la France (NGF) (a), the background shows the reference LiDAR DTM; DTM of difference (in $\mathrm{m}$ ) of the interpolated DTM and reference DTM created from LiDAR data (b) (a shaded relief map is shown behind maps for context); b1: MO method, b2: Kriging interpolation, b3: Delaunay triangulation TIN.

\subsection{Comparison with other interpolation methods}

Such interpolation methods using the proposed in-situ morphologicallybased method would correspond roughly to the "bar and chute outline" as introduced by Heritage et al. (2009) using different usual routines for interpollation. 
There are many routines available for interpolation in GIS software like SurferC) (Surfer, 2002), which could be used to do the interpolation. For the aim of the current study, a simple Kriging algorithm and the Delaunay triangulation with linear interpolation (TIN) algorithm were used to generate DTMs based on the same degraded data from LiDAR as the one used for the MO method. These two methods are widely tested, documented, and commonly used (Desmet, 1997; Erdogan, 2009; Merwade, 2009). Although, the TIN approach supports the use of breaklines, it is limited to the major ones reflecting obvious breaks in slope and large features (Heritage et al., 2009). Generally, these breaklines are directly measurable in the field and are easy to identify by visualizing the original scatter of points. Therefore, there is no need for cross section views to define major breaklines. However, in the current methodology, a wide use of breaklines is desirable for more accuracy. This cannot be done without cross-section visualization. Consequently, for the sake of this comparison, the TIN approach was constructed without breaklines as none of them can be easily defined from the scatter of points shown in Fig. 1.

A structural difference in the estimated value of the elevation can be clearly seen in Fig. 6b2, which gives the DTM of difference between the simple Kriging interpolation and the reference DTM. The spatial distribution of the error magnitude shows some redundancy. The prediction error is very high at locations where no measurement points are chosen in the degradation process. The Kriging method is dependent on the spatial lag and spatial distribution of point measurements and is not adapted to irregular distribution. By examining Fig. 6b2, it can be seen that the two parts delimited by the bar edge show an opposite nature of error: (i) an overestimation on the steep part delimited by the bar edge and the main channel (ii) an underestimation of the flat part on the bar surface located at the left part of the bar edge. The same effect is observed also on the limits of the two banks of the secondary channel where there is a change in the transversal slope. For both cases, this can be explained by the weight given for the nearby points by the Kriging method. Therefore, to interpolate points in the steep part, the Kriging method uses some points located at the flat zone on the top of the gravel bar that has a higher elevation. Thus, the inference of the two morphologically inhomogeneous zones results in an inaccurate DTM. The importance of weight given to nearby points in Kriging even though slope is very high is corrected in the MO method by imposing breaklines. The usefulness of breaklines is dividing the river bed into different zones where each zone has 
the same transverse slope trend and then interpolation is done following the longitudinal direction without interfering with the adjacent incoherent zones.

The TIN method was used to reproduce the DTM from the point dataset at the same grid size as the reference DTM. Figure $6 \mathrm{~b} 3$ plots the DTM of difference between the two cited DTMs. The results show a less systematic loss of information than for the Kriging method, but the TIN generated DTM is still less accurate than the MO DTM. Since the TIN method uses just three points to calculate the elevation at a certain location, and, therefore, gives a large weight to these three considered points, the DTM error is less sparse in space and mainly tends to underestimate the real elevation. Nevertheless, the predicted error is clustered and spatially correlated. It is directly linked to steep slopes at the edge of the gravel bar and transverse channels. As can be seen in Fig. 6b3, the TIN method produces an underestimation of the real elevation especially at the banks of the transverse channels where curvature is large. A possible explanation is the interference of points located at the transverse channel banks and their flat beds.

\subsection{Validation with data from a topographic campaign}

Degradation of data to a lower density allows assessment of the dependency relation between interpolation method, rendered DTM quality, and data density using three interpolation methods: MO, Kriging, and TIN. Such analysis allows determination of optimal point measurement density and location. Thus, the same degradation methodology was applied for the topographic campaign of October 2011. The repeated field surveys made since 2007 on the gravel bar have an average data density varying around 0.035 points $/ \mathrm{m}^{2}$ whereas the 2011 dataset used for the previous example has a density equal to 0.060 points $/ \mathrm{m}^{2}$. The degradation was done in regard to the MO recommendations and hypothesis; therefore, particular attention was given to keep data points that describe different topographic characteristics of the reach as for a normal survey campaign of lower density. The final density is equal to 0.036 points $/ \mathrm{m}^{2}$. A comparison with other interpolation methods is necessary to assess the efficiency of the MO method for both reference data (LiDAR) and typical field survey data.

The results are summarized in Table 1. The three DTMs produced by the three interpolation methods exhibit low global error estimation despite the low density of the measurement points used. This confirms the conclusion made by Heritage et al. (2009) who studied the importance of survey strategy and concluded that DTM error is strongly influenced by the location of survey 
Table 1. Comparison of error estimation for MO, Kriging, and TIN interpolation methods using two different data densities and sources obtained by degrading the May 2010 LiDAR dataset and the dataset obtained from the October 2011 topographic campaign. ( $E_{\mathrm{ma}}$ is the mean absolute error and $\sigma$ is the standard deviation).

\begin{tabular}{c|lrrrrr} 
& Method & $\begin{array}{c}\text { Used } \\
\text { points }\end{array}$ & $\begin{array}{r}\text { Check } \\
\text { points }\end{array}$ & $\begin{array}{l}\text { Mean } \\
\text { error }(\mathrm{m})\end{array}$ & $\begin{array}{r}E_{m a} \\
(\mathrm{~m})\end{array}$ & $\begin{array}{r}\sigma \\
(\mathrm{m})\end{array}$ \\
\hline \multirow{3}{*}{ LiDAR data } & MO & 669 & 58546 & -0.010 & 0.048 & 0.072 \\
& Kriging & 669 & 58546 & 0.019 & 0.083 & 0.126 \\
& TIN & 669 & 58546 & -0.014 & 0.077 & 0.118 \\
\hline \multirow{3}{*}{ Topographic data } & MO & 1023 & 674 & 0.012 & 0.079 & 0.106 \\
& Kriging & 1023 & 674 & -0.032 & 0.148 & 0.225 \\
& TIN & 1023 & 674 & 0.005 & 0.101 & 0.162
\end{tabular}

points relative to the morphology and that a strong relation exists between local surface topographic variation and DTM error. A DTM of about 58, 000 grid points is reproduced by the MO method with a global mean absolute error $E_{m a}=0.048 \mathrm{~m}$ just by using 669 adequately chosen points. Thus, it can be concluded that there is an optimal data density producing enough information about the complex morphology, which is essential for the field survey strategy. By examining error estimation for the same dataset source, the improvement of DTM accuracy made by the MO method can be seen. In fact, for both dataset sources, the MO method has the lowest $E_{m a}$ and $\sigma$ values.

\section{Error estimation}

\subsection{GWR application}

The GWR method (see Eq. 3) applied to the reference LiDAR data yields the correlation coefficients shown in Fig. 7. It can be seen that all three parameters vary in space. In particular, the coefficients are sensitive to the distance to the measured points.

The results of the GWR application are shown in Fig. 8a. The predicted error magnitude and spatial dispersion are similar to the real calculated DTM error shown in Fig. 6b. The GWR model results yield almost the same value of minimal error and buffer zones where error magnitude is high. The optimum global adjusted coefficient of determination $\left(R^{2}\right)$ is found to be equal 


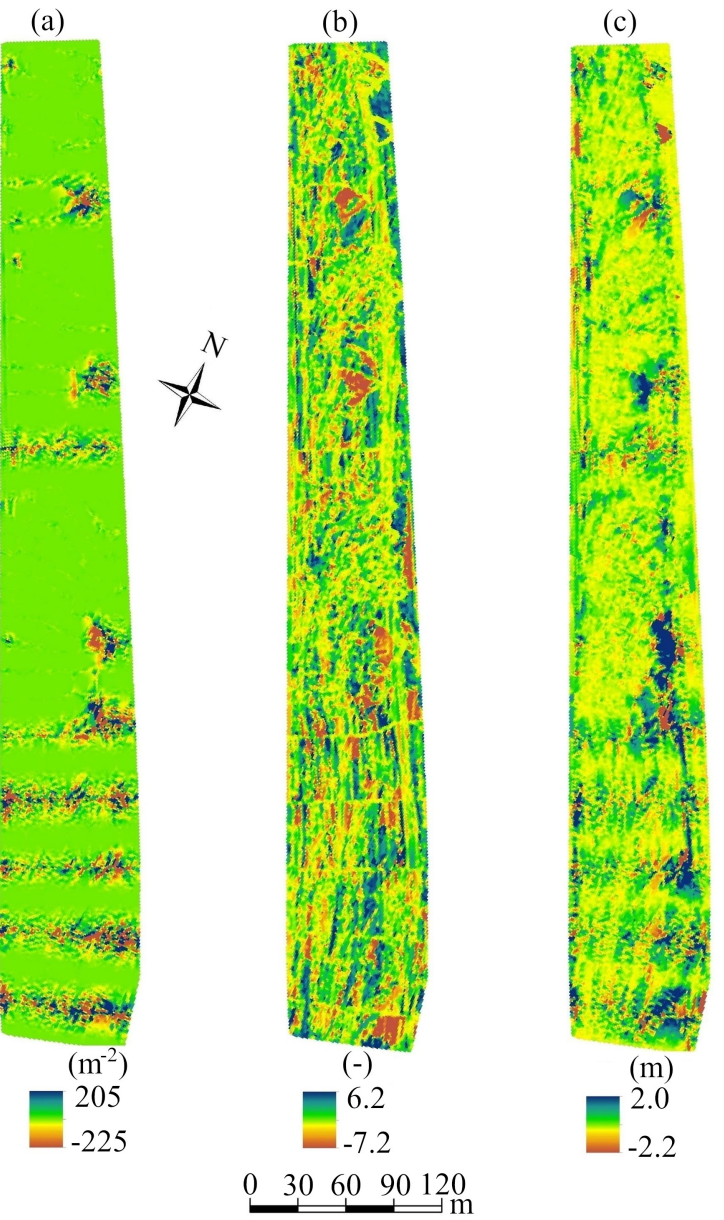

Fig. 7. Spatial distribution of inverse squared distance correlation coefficient $\alpha_{1}\left(x_{i}\right)$ (in $\mathrm{m}^{-2}$ ) (a), slope correlation coefficient $\alpha_{2}\left(x_{i}\right)$ (dimensionless) (b), and intercept term $\alpha_{0}\left(x_{i}\right)$ (in $\mathrm{m}$ ) (c) defined in Eq. 3

0.95 which is a good improvement of the OLS regression model where $R^{2}$ is only equal to 0.14 . The adjusted $R^{2}$ is equal to 0.90 confirm the goodness of to the predicted error. The improvement of the local autocorrelation model (GWR) in comparison with the global model (OLS) is expected. In fact, by construction, the MO method tends to limit, as much as possible, the variation of the geomorphometric parameters and especially the slope. Thus, by dividing the reach into homogeneous zones where slope is constant and imposing the non-inference of points from two different zones in the regression algorithm, the MO method reduces the incidence of slope variation on 
predicted elevation and therefore reduces the correlation of elevation error to slope. The MO method allows linear interpolation based on the nearest points belonging to the same zone delimited by two breaklines, hence, the local autocorrelation performs better than the global autocorrelation.

\subsection{Spatial error estimation}

The aim of this error study consists in trying to develop a simple uncertainty model that includes the true error estimation whereby error for other field surveys where not enough data are available to compute global error statistic parameters can be evaluated. Therefore, the spatial GWR model is considered as a starting point.

- First, a database is constructed using the different values of the two grids of correlation coefficients $\alpha_{1}\left(x_{i}\right)$ and $\alpha_{2}\left(x_{i}\right)$ and the intercept term $\alpha_{0}\left(x_{i}\right)$ with their relative inverse squared distance and slope obtained thanks to the extensive LiDAR data (see Section 5.1);

- Second, slope and inverse squared distance are computed for every point $x_{j}$ defining a given DTM;

- Third, for every $x_{j}$ the database is examined for the point $x_{i, j}$ for which the absolute difference between inverse square distances $\left(d\left(x_{i, j}\right)^{-2}-\right.$ $\left.d\left(x_{j}\right)^{-2}\right)$ and slopes $\left(\left\|\overrightarrow{\operatorname{grad}}\left(x_{i, j}\right)\right\|-\left\|\overrightarrow{\operatorname{grad}}\left(x_{j}\right)\right\|\right)$ are minimal;

- Finally, the correlation coefficients of $x_{i, j}$ are assigned to $x_{j}$ and are used to calculate error at the point $x_{j}$. The final product is a grid to which the device systematic error is added (see section 3.1).

Consequently a spatial uncertainty model can be produced for each DTM, which defines an upper boundary of the DTM error. It should be noted that the proposed method to determine the uncertainty due the interpolation method is indirect and so has an uncertainty by itself. As a first approximation, this uncertainty is neglected. Another limitation of this calculation is that the data base built for the three coefficients of Eq. 3 could be partly site-dependent although it presents a wide collection of slopes and inverse squared distances. It would be interesting to check this data base on a different site. An application of this error estimation to the field campaign of October 2011 is shown in Fig. 8(b). It can be seen that prediction error is higher in steep locations, especially at the bar margins. On the contrary, 
prediction error is relatively low in flat locations (bar tail). Moreover, error appears clearly sensitive to the data density (upstream part of the main channel, see Fig. 6 for the data density). It is also interesting to see that the roughness error, which varies from 1 to $100 \mathrm{~mm}$ (see Eq. 2 and Fig. 5) can represent up to $50 \%$ of the error in zones with hih data density.

(a)

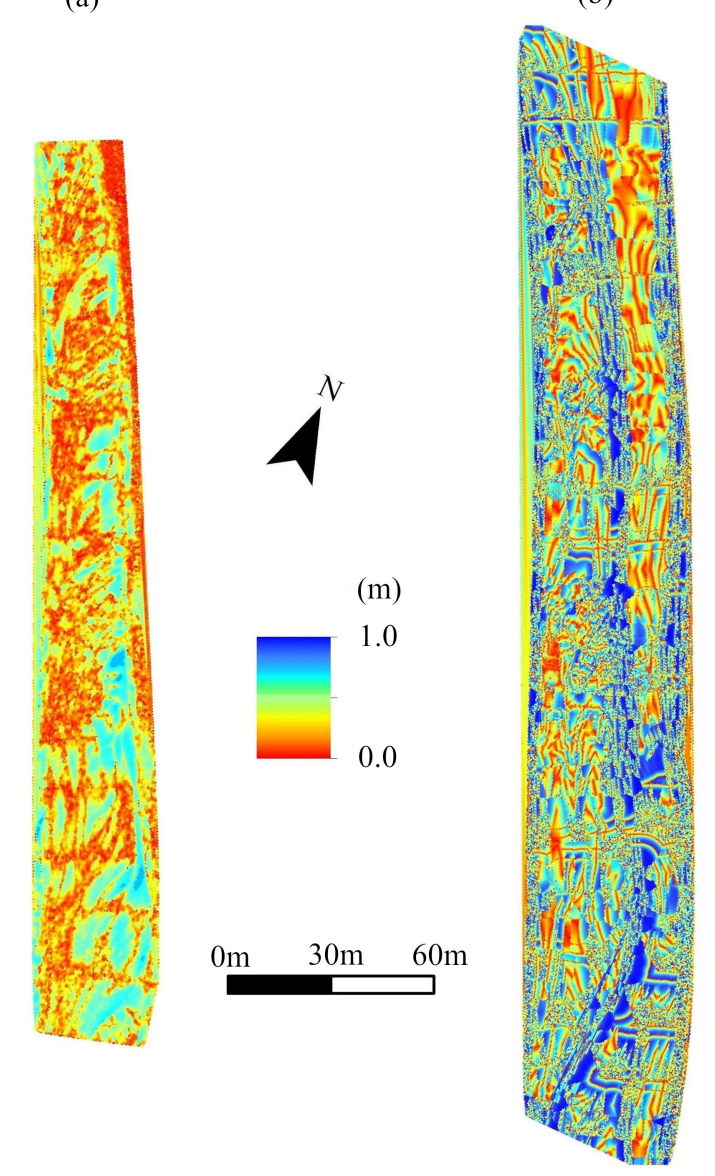

Fig. 8. Spatial distribution of the predicted error (in $\mathrm{m}$ ) by the GWR model applied to the reference LiDAR data (a) and applied to the field campaign of October 2011 (b) (reach size is larger for (b) because area under water is excluded in the LiDAR data (a) but included in the field campaign application (b)).

Considering the volume error, for the reference (LiDAR) data, a direct computation of this value is done by estimating the volume of the DTM of difference between interpolated and reference DTMs (See Table 1). The 
directly measured volume of the error is $706 \mathrm{~m}^{3}$. The predicted volume error using the GWR is very similar and equal $695 \mathrm{~m}^{3}$. Knowing that the reach surface is $14,644 \mathrm{~m}^{2}$, if a uniform error is considered equal to the $E_{\mathrm{ma}}$, therefore, the volume error is equal to $703 \mathrm{~m}^{3}$, which is in accordance with the direct measurement in contrast with $\sigma$, which yields larger volume errors, respectively, equal to $1,054 \mathrm{~m}^{3}$ and $1,069 \mathrm{~m}^{3}$. Hence, $E_{\mathrm{ma}}$ is more suitable for volume error computation. In general, measurement errors are centimetric $\left(\delta_{\mathrm{m}}=0.02 \mathrm{~m}\right.$ here) whereas roughness errors could lead to values up to $0.05-0.10 \mathrm{~m}$ in gravel bed rivers (see Eq. 2). Interpollation errors can be decimetric especially in steep locations with low data density. As a consequence, generally $\delta_{\mathrm{m}}(z) \ll \delta_{\mathrm{r}}(z) \ll \delta_{\mathrm{p}}(z)$ and so most uncertainty in the estimation of a volume of the DTM come from the interpollation in case of densities lower than 0.05 points $/ \mathrm{m}^{2}$.

\section{Conclusions}

This current paper describes the development of a morphologically oriented survey strategy and interpolation methods to reproduce the complex morphological characteristics of rivers as well as an estimation of the uncertainties. The MO method is applied and validated on a river reach including a gravel bar and secondary channels.

The following general conclusions can be drawn from the study:

- The field survey strategy is optimized to identify the global and local topographic characteristics of the river bed by describing the river reach thanks to a set of cross sections and breaklines at global and local scales. This morphological based method allows all major breaks in slope and all major directions of water flow to be described depending on the water discharge.

- An exact interpolation method (e.g., true measured points are retrieved in the final grid) is developed. It is based on linear longitudinal interpolation over homogeneous parts of surface topography defined by breaklines.

- The MO method is validated using a DTM of reference derived from LiDAR data. It is shown that the MO method produced DTMs which exhibit low global error. Larger uncertainty is observed in very specific and isolated locations corresponding to singular boulders or small breaches. 
- Uncertainty estimation is calculated using a method assessing spatial variability of error related to the distance to the closest measured point and the slope, and then combined with a bed roughness map.

Although the proposed methodology requires more time to process data that the one proposed by Heritage et al. (2009) (because of the need to define two sets of breaklines defining both main longitudinal features and transverse features; two-step interpollation procedure instead of one step), it is eventually more accurate and robust.

Finally, besides the recent advancement in remotely sensed data acquisition, the use of ground-based surveys still is valuable especially for zones under water where dense, accurate, and spatially distributed information cannot be acquired. Ground-based surveys give enough accuracy for a wide range of applications with a relatively small cost/time resource. Groundbased surveys must focus on local topographic features and breaks in slope and must be adapted to interpolation methods guided by breaklines. When developing a field survey strategy, a surveyor should remember that one cannot measure all terrain configurations. Hereby the MO method appears as one simple quick and accurate method to reproduce real elevation based on a limited amount of data. But it is obvious, regardless the cost of data acquisition, that a combination of data sources (structure-from-motion, LiDAR, laser scanning, etc.) will produce much more accurate and detailed DTMs.

The definition of breaklines, which are required to reproduce transverse channels, could also be developed thanks to automatic detection. An automatic detection of breaklines could be of great interest for applying the MO method to more complicated cases. In the case of braided rivers, for example, a large number of breaklines would need to be defined including bifurcations and merging. Automatic detection of breaklines requires however a dense data set, which, thus, implies a combination between the proposed methodology and LiDAR or terrestrial laser scan acquisition (Schäppi et al., 2010).

\subsection{Acknowledgements}

This study was supported by Irstea as well as the Rhône-Alpes region through the CMIRA ExploraPro financial support. We would like to thank Charles Melching for his comments and inputs to improve the paper. 


\section{References}

Aggett, G. R., \& Wilson, J. P. (2009). Creating and coupling a highresolution DTM with a 1-D hydraulic model in a GIS for scenario-based assessment of avulsion hazard in a gravel-bed river. Geomorphology, 113, 21-34. doi:10.1016/j.geomorph.2009.06.034.

Bangen, S., Wheaton, J., Bouwes, N., Jordan, C., Volk, C., \& Ward, M. B. (2014). Crew variability in topographic surveys for monitoring wadeable streams: A case study from the Columbia River basin. Earth Surface Processes 65 Landforms, 39, 2070-2086. doi:10.1002/esp.3600.

Brasington, J., Langham, J., \& Rumsby, B. (2003). Methodological sensitivity of morphometric estimates of coarse fluvial sediment transport. Geomorphology, 53, 299-316. doi:10.1016/S0169-555X(02)00320-3.

Brasington, J., Rumsby, B. T., \& McVey, R. A. (2000). Monitoring and modelling morphological change in a braided gravel-bed river using high resolution GPS-based survey. Earth Surface Processes 8 Landforms, 25, 973-990. doi:10.1002/1096-9837(200008) 25:9<973: :AID-ESP111>3.0. $\mathrm{CO} ; 2-\mathrm{Y}$.

Camenen, B., Le Coz, J., Paquier, A., \& Lagouy, M. (2010). An estimation of gravel mobility over an alpine river gravel bar (Arc en Maurienne, France) using PIT-tag tracers. In A. Dittrich, K. Koll, J. Aberle, \& P. Geisenhainer (Eds.), River Flow, Proc. 5th Int. Conf. on Fluvial Hydraulics (pp. 953960). Braunschweig, Germany.

Carley, J., Pasternack, G., Wyrick, J. R., Barker, J. R., Bratovich, P. M., Massa, D. A., Reedy, G. D., \& Johnson, T. R. (2012). Significant decadal channel change 58-67 years post-dam accounting for uncertainty in topographic change detection between contour maps and point cloud models. Geomorphology, 179, 71-88. doi:10.1016/j.geomorph.2012.08.001.

Carlisle, B. H. (2005). Modelling the spatial distribution of DEM error. Transactions in GIS, 9, 521-540. doi:10.1111/j.1467-9671.2005. 00233.x.

Desmet, P. J. J. (1997). Effects of interpolation errors on the analysis of DEMs. Earth Surface Processes \&6 Landforms, 22, 563-580. doi:10.1002/ (SICI) 1096-9837(199706) 22:6<563: : AID-ESP713>3.0.C0;2-3. 
Erdogan, S. (2009). A comparison of interpolation methods for producing digital elevation models at the field scale. Earth Surface Processes $\&$ Landforms, 34, 366-376. doi:\{10.1002/esp.1731\}.

Erdogan, S. (2010). Modelling the spatial distribution of DEM error with geographically weighted regression: An experimental study. Computers 8 Geosciences, 36, 34-43. doi $10.1016 /$ j.cageo.2009.06.005.

Fotheringham, A. S., Brunsdon, C., \& Charlton, M. (2002). Geographically weighted regression: The analysis of spatially varying relationships. New York, USA: John Wiely \& Sons.

French, J. R., \& Clifford, N. J. (2000). Hydrodynamic modelling as a basis for explaining estuarine environmental dynamics: Some computational and methodological issues. Hydrological Processes, 14, 2089-2108. doi:10.1002/1099-1085(20000815/30)14:11/12<2089: : AID-HYP56>3.0.CO;2-L.

Fuller, I. C., Large, A. R. G., Charlton, M. E., Heritage, L., \& Milan, D. J. (2003). Reach-scale sediment transfers: an evaluation of two morphological budgeting approaches. Earth Surface Processes \& Landforms, 28, 889-903. doi:10.1002/esp.1011.

Heritage, G. L., Milan, D. J., Large, A. R. G., \& Fuller, I. C. (2009). Influence of survey strategy and interpolation model on DEM quality. Geomorphology, 112, 334-344. doi:\{10.1016/j.geomorph.2009.06.024\}.

Horritt, M. S., \& Bates, P. D. (2002). Evaluation of 1D and 2D numerical models for predicting river flood inundation. Journal of Hydrology, 268, 87-99. doi:\{10.1016/S0022-1694(02)00121-X\}.

Jaballah, M. (2013). Alternate bar morphodynamics in an engineered mountainous river. Ph.D. thesis Claude Bernard University, Lyon 1, Irstea Hydrology-Hydraulics Research Unit Lyon, France. 196 p.

Jodeau, M. (2007). Morphodynamique d'un banc de galets en rivière aménagée lors de crues [Gravel bar morphodynamics in an engineered river during high flow events]. Ph.D. thesis Claude Bernard University, Lyon 1, , Cemagref Hydrology-Hydraulics Research Unit Lyon, France. 205p. (in French). 
Keim, R. F., Skaugset, A. E., \& Bateman, D. S. (1999). Digital terrain modeling of small stream channels with a total-station theodolite. Advances in Water Resources, 23, 41-48. doi:10.1016/S0309-1708(99)00007-X.

Lane, S. N. (1998). Hydraulic modelling in hydrology and geomorphology: a review of high resolution approaches. Hydrological Processes, 12, 1131-1150. doi 10.1002/(SICI) 1099-1085(19980630) 12:8<1131: : AID-HYP611>3.0.CO;2-K.

Lane, S. N., \& Ferguson, R. I. (2005). Modelling reach-scale fluvial flows. In P. D. Bates, S. N. Lane, \& R. I. Ferguson (Eds.), Computational fluid dynamics, applications in environmental hydraulics (pp. 217-279). New York, USA: John Wiley and Sons, Ltd.

Legleiter, C. J. (2012). Remote measurement of river morphology via fusion of LiDAR topography and spectrally based bathymetry. Earth Surface Processes \& Landforms, 37, 499-518. doi:10.1002/esp.2262.

Legleiter, C. J., \& Kyriakidis, P. C. (2008). Spatial prediction of river channel topography by kriging. Earth Surface Processes \& Landforms, 33, 841867. doi:10.1002/esp.1579.

Legleiter, C. J., Kyriakidis, P. C., McDonald, R. R., \& Nelson, J. M. (2011). Effects of uncertain topographic input data on two-dimensional flow modeling in a gravel-bed river. Water Resources Research, 47, 1-24. doi:\{10.1029/2010WR009618\}.

Merwade, V. (2009). Effect of spatial trends on interpolation of river bathymetry. Journal of Hydrology, 371, 169-181. doi:10.1016/j. jhydrol.2009.03.026.

Merwade, V., Cook, A., \& Coonrod, J. (2008). GIS techniques for creating river terrain models for hydrodynamic modeling and flood inundation mapping. Environmental Modelling and Software, 23, 1300-1311. doi:10.1016/j . envsoft.2008.03.005.

Milan, D. J., Heritage, G. L., Large, A. R. G., \& Fuller, I. C. (2011). Filtering spatial error from DEMs: Implications for morphological change estimation. Geomorphology, 125, 160-171. doi:\{10.1016/j.geomorph.2010.09. 012\}. 
Miroslaw-Swiatek, D., Michalowski, R., Szporak-Wasilewska, S., Ignar, S., \& Grygoruk, M. (2016). Unraveling uncertainties of water table slope assessment with dgps in lowland floodplain wetlands. Environmental Monitoring \& Assessment, 188, 1-11. doi:10.1007/s10661-016-5642-3.

Pénard, L. (2010). FudaA Modeleur - Secma guide rapide en vue de l'utilisation de RUBAR 20 [FUDAA MODELEUR - SECMA short user's manual]. Internal Report, Irstea, Lyon, France (in French).

Schäppi, B., Perona, P., Schneider, P., \& Burlando, P. (2010). Integrating river cross section measurements with digital terrain models for improved flow modelling applications. Computers \& Geosciences, 36, 707716. doi:10.1016/j.cageo.2009.12.004.

Surfer (2002). Surfer 8. Contouring and 3D Surface Mapping for Scientist and Engineers. User's Guide. Technical Report Golden Software, Inc.

Wechsler, S. P. (2007). Uncertainties associated with digital elevation models for hydrologic applications: a review. Hydrology and Earth System Sciences, 11, 1481-1500. doi:10.5194/hess-11-1481-2007.

Westoby, M., Brasington, J., Glasser, N., Hambrey, M., \& Reynolds, J. (2012). 'structure-from-motion' photogrammetry: A low-cost, effective tool for geoscience applications. Geomorphology, 179, 300-314. doi:10. 1016/j.geomorph.2012.08.021.

Wheaton, J. M., Brasington, J., Darby, S. E., \& Sear, D. A. (2010). Accounting for uncertainty in DEMs from repeat topographic surveys: improved sediment budgets. Earth Surface Processes \& Landforms, 35, 136-156. doi:10.1002/esp.1886.

Wolman, M. G. (1954). A method of sampling coarse river-bed material. Transactions of the American Geophysical Union, 35, 951-956. doi:10. 1029/TR035i006p00951.

Woodget, A. S., Carbonneau, P. E., Visser, F., \& Maddock, I. P. (2015). Quantifying submerged fluvial topography using hyperspatial resolution UAS imagery and structure from motion photogrammetry. Earth Surface Processes $\&$ Landforms, 40, 47-64. doi:10.1002/esp.3613. 